\title{
Finite-temperature molecular-dynamics study of unstable stacking fault free energies in silicon
}

\section{Citation}

Koning, M. de, A. Antonelli, Martin Z. Bazant, Efthimios Kaxiras, and J. F. Justo. 1998. "FiniteTemperature Molecular-Dynamics Study of Unstable Stacking Fault Free Energies in Silicon." Physical Review B 58 (19): 12555-58. https://doi.org/10.1103/physrevb.58.12555.

\section{Permanent link}

http://nrs.harvard.edu/urn-3:HUL.InstRepos:41384075

\section{Terms of Use}

This article was downloaded from Harvard University's DASH repository, and is made available under the terms and conditions applicable to Other Posted Material, as set forth at http:// nrs.harvard.edu/urn-3:HUL.InstRepos:dash.current.terms-of-use\#LAA

\section{Share Your Story}

The Harvard community has made this article openly available.

Please share how this access benefits you. Submit a story.

Accessibility 


\title{
Finite temperature molecular dynamics study of unstable stacking fault free energies in silicon
}

\author{
M. de Koning and A. Antonelli \\ Instituto de Física Gleb Wataghin, Universidade Estadual de Campinas, \\ Unicamp 13083-970, Campinas, São Paulo, Brazil \\ Martin Z. Bazant \\ Department of Mathematics, \\ Massachusetts Institute of Technology, Cambridge, MA 02139 \\ Efthimios Kaxiras \\ Department of Physics and Division of Applied Sciences, \\ Harvard University, Cambridge, MA 02138 \\ J.F. Justo \\ Instituto de Física da Universidade de São Paulo, \\ CP 66318, CEP 05315-970 São Paulo - SP, Brazil
}

\begin{abstract}
We calculate the free energies of unstable stacking fault (USF) configurations on the glide and shuffle slip planes in silicon as a function of temperature, using the recently developed Environment Dependent Interatomic Potential (EDIP). We employ the molecular dynamics (MD) adiabatic switching method with appropriate periodic boundary conditions and restrictions to atomic motion that guarantee stability and include volume relaxation of the USF configurations perpendicular to the slip plane. Our MD results using the EDIP model agree fairly well with earlier first-principles estimates for the transition from shuffle to glide plane dominance as a function of temperature. We use these results to make contact to brittle-ductile transition models.
\end{abstract}

PACS number:02.70.Ns,65.50.+m,62.20.Mk,61.72.-y

The physics describing the behavior of extended defects at the microscopic level and its relation to macroscopic mechanical properties of materials, which are of fundamental importance to technology, have been subject of investigation for many years. An example of intriguing macroscopic behavior is the brittle-ductile transition (BDT), corresponding to a change in the state of the system from a brittle, easily fractured, into a ductile, tough substance that can easily undergo plastic deformation. Silicon is a material in which the BDT is particularly spectacular, taking place over a very narrow temperature range of only a few degrees $K$, th a a critical temperature near $873 \mathrm{~K}$. Brittle or ductile behavior is related to the response of a sharp crack tip to external loading: Brittleness is typically associated with easy crack propagation, whereas ductility is characterized by blunting of the crack tip through the emission of dislocations. The microscopic mechanisms behind the crack tip response are related to the ability of the solid to nucleate and emit dislocations from the loaded crack tip.3

While complex atomistic processes such as dislocation nucleation and mobility cannot easily be captured by simple phenomenological models, certain features of the structure of Si may be related to the abruptness of the BPT in this material. In silicon two distinct sets of closely packed $\{111\}$ slip planes, called "glide" and "shuffle" setsB, are relevant for dislocation nucleation and slip. Dislocations nucleated on the shuffle set are relatively narrow making the resistance to dislocation motion, the so-called Peierls stress, relatively high and their mobility low. On the glide set the mobility properties are different. Due to the splitting of dislocations into partials on this set, the corresponding Peierls stresses are smaller and the dislocation mobility is higher. In this sense, the abruptness of the BDT in silicon may be associated with a sudden change in the dominance of one set over the other. In order to characterize such a change, a theoretical model is required that can link the processes of dislocation nucleation and motion to simple, material-specific quantities which can be calculated afcurately.

Recent theoretical work by Rice and collaborator 10 developed such a model of dislocation nucleation at a crack tip based on a continuum elasticity approach and the Peierls stress concept. From this analysis, the so-called unstable stacking energy $\gamma_{u s}$ was found to be a measure for the resistance to dislocation nucleation at a crack tip (we refer to this below as the dislocation nucleation criterion). The unstable stacking energy, which corresponds to the unstable stacking fault (USF) configuration 4.5 is defined as the lowest energy barrier that needs to be crossed when one half of a perfect crystal slips, on a dislocation nucleation plane of interest, relative to the other half, completing a total displacement equal to one lattice repeat vector. 
The calculations performed by Kaxiras and Duesbery 1 and Juan and Kaxiras 5 , based on first principles Density Functional Theory techniques within the Local Density Approximation (DFT-LDA), provided accurate values for $\gamma_{u s}$ on the shuffle and glide planes of Si. Through the use of Vineyard's Transition State Theory (TST), 0 these zero temperature results were extended to finite temperature and pressure conditions 4.6 This approach, while illustrating the basic idea of an abrupt transition from shuffle to glide set dominance as a function of temperature, is theoretically limited, because it neglects real dynamics of atoms on either side of the slip plane and it relies on mapping the system to an oversimplified two-dimensional model. It is therefore desirable to include finite temperature effects in an explicit manner. To this end, finite temperature molecular dynamics (MD) simulation of the USF configurations would represent an interesting improvement. Within such a context the application of a finite temperature firstprinciples MD method would be most appropriate. However, as long as the present computational limitations of such techniques inhibit their application to larger systems over wide temperature ranges, an approximate approach based on empirical models remains the only alternatige With the development of the novel empirical Environment Dependent Interatomic Potential (EDIP) for silicon, 1213 the application of such an approach seems now feasible. As opposed to other empirical models, 10.11 EDIP captures with adequate realism several important stable, metastable and saddle-point configurations, including the energetics of generalized stacking faults and dislocation cores, and promises to be very useful for the description of the dynamics of USF configurations.

In this brief report we address the question of the influence of finite temperature effects on the dislocation nucleation criterion, through an explicit calculation of the free energies associated with the USF configurations on the glide and shuffle sets as a function of temperature. For this purpose we employ the MD adiabatic switching method which is based on the simulation of thermodynamically reversible processes and enables the efficient and quantitatively reliable determination of thermal quantities including all anharmonic effects. $14-6$ We use the EDIP model for the description of the interatomic forces in the MD simulations and compare our results to DFT-LDA results.

Two specific technical issues are involved in the MD simulation of USF configurations. The first concerns the definition of the two atomic USF configurations (shuffle and glide) within MD cells. For this purpose, the choice of an orthogonal coordinate system formed by axes parallel to [111], [101] , and [12̄1] directions is most appropriate. The glide and shuffle USF configurations are defined by the slip displacement vectors $\frac{1}{12}[1 \overline{2} 1]$ and $\frac{1}{4}[10 \overline{1}]$ (in units of the lattice parameter) 4 国 respectively, which describe the relative displacement of the two atomic blocks adjacent to the crystal slip plane under consideration. These properties are implemented by adding the corresponding slip displacement vectors on the periodic boundary repeat vectors in the [111] direction of the computational cells.

The second issue is related to the fact that the USF configurations are intrinsically unstable. Due to the state of slip of these configurations, considerable shear stresses appear in the system, which tend to relax through shear strain. In order to prevent this relaxation during the MD simulations, the motion of the atoms in the two planes immediately adjacent to the slip planes is restricted to the [111] direction, perpendicular to the shear stresses.

The size of the computational cells utilized for the simulation of the USF configurations is choosen such that the interaction between the periodic images is negligibly small. To this end, the number of atoms for both the glide and shuffle USF configurations was fixed at 648, divided in 36 atomic (111) planes. In order to allow for volume relaxation perpendicular to the slip planes, $\gamma_{u s}$ (including atomic relaxation) is evaluated at several volumes below and above the ideal volume of bulk silicon. For the glide set, the minimum value of $\gamma_{u s}$ is obtained for a perpendicular expansion $\Delta z_{\text {glide }}=0.35 \AA$, while the minimum value of $\gamma_{u s}$ for the shuffle set USF configuration is obtained for a contraction $\Delta z_{\text {shuffle }}=-0.22 \stackrel{\circ}{A}$.

TABLE I. Static $(0 K)$ unstable stacking energy $\gamma_{u s}$ for the $\{111\}$ shuffle and glide set in silicon at various levels of relaxation. All values are in $\mathrm{J} \mathrm{m}^{-2}$. The DFT results were taken from Juan and Kaxiras (Ref. 6).

\begin{tabular}{c|c|c}
\hline \hline & Shuffle set & Glide set \\
\hline No relaxation & & 2.51 \\
\hline DFT-LDA & 1.84 & 3.28 \\
EDIP & 1.98 & 2.02 \\
\hline Atomic relaxation & & 1.89 \\
\hline DFT-LDA & 1.81 & \\
EDIP & 1.28 & 1.91 \\
\hline Atomic+volume relaxation & & 1.86 \\
\hline DFT-LDA & 1.67 & 1.04 \\
EDIP & & \\
\hline \hline
\end{tabular}


Table il shows several $0 K \gamma_{u s}$ values characterized by different levels of relaxation, as obtained with EDIP and DFTLDA.D Although EDIP correctly predicts $\gamma_{u s}$ to be higher for the glide set than for the shuffle set, the quantitative discrepancies with DFT-LDA are significant. The consequences of these discrepancies will be discussed further below. Despite the quantitative differences, EDIP successfully captures the qualitative trends predicted by DFT-LDA for the effects of relaxation on $\gamma_{u s}$. Both approaches predict the influence of atomic relaxation to be larger on the glide set, and that volume relaxation is more pronounced on the shuffle set.

In order to evaluate the free energies of the USF configurations, a series of MD adiabatic switching simulations is performed for several temperatures between 200 and $1600 \mathrm{~K}$. In these simulations, the interacting silicon atoms are transformed into identical harmonic oscillators, under $(N, V, T)$ conditions. 15 . 16 The equilibrium positions of the oscillators are centered at the equilibrium positions of the silicon atoms in the USF configurations and all oscillators have the same characteristic frequency. Effects of thermal expansion are taken into account using lattice constants determined from standard $(N, P, T)$ simulations for bulk silicon.

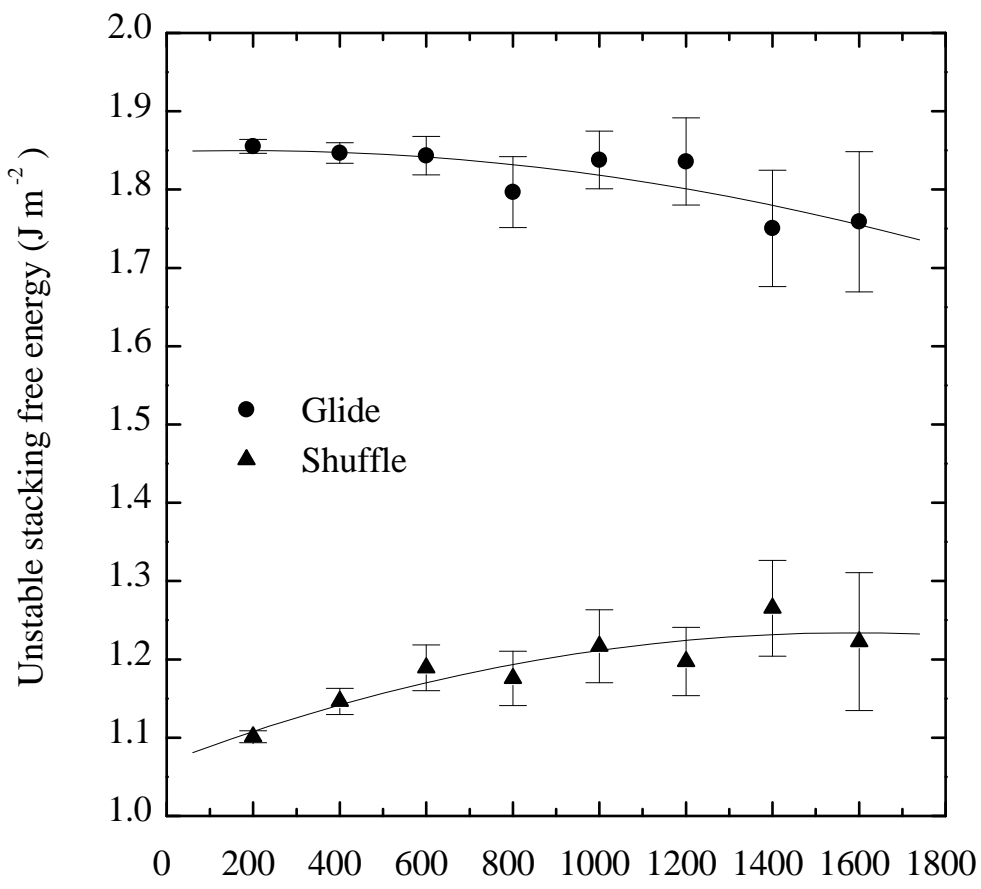

$\mathrm{T}(\mathrm{K})$

FIG. 1. USF Helmholtz free energies as a function of temperature, for the glide and shuffle sets in silicon at zero pressure. 


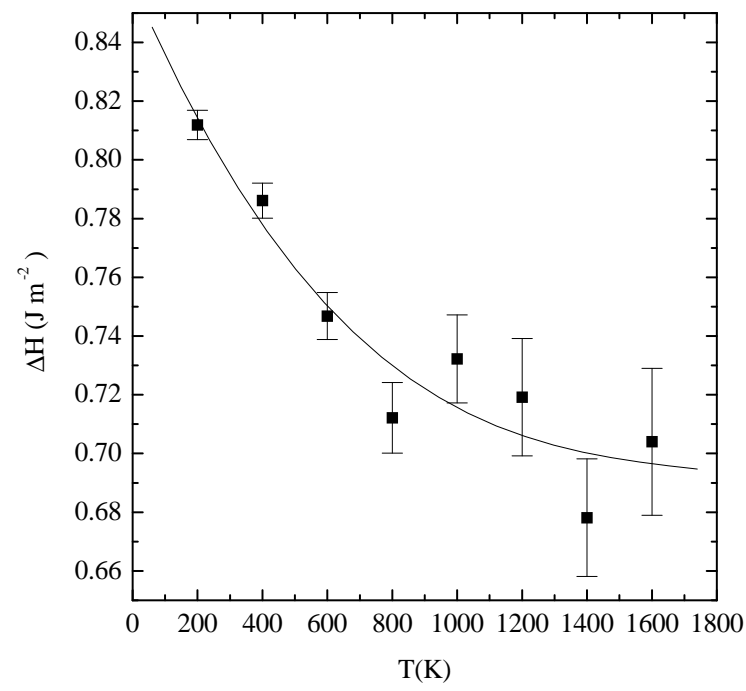

FIG. 2. Zero pressure enthalpy difference between the glide and shuffle USF configurations as a function of temperature.

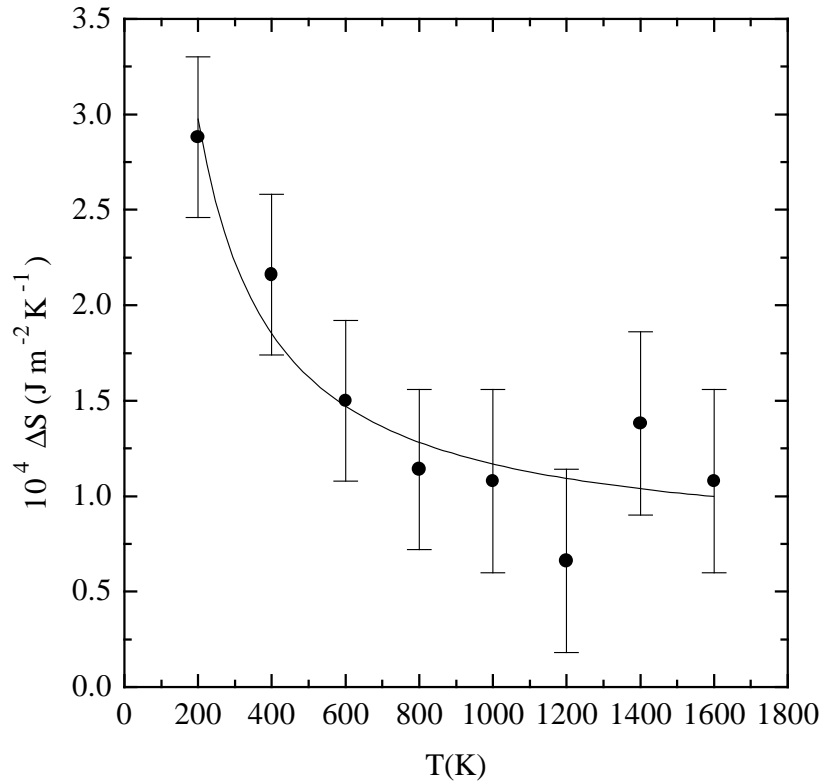

FIG. 3. Entropy difference between the glide and shuffle USF configurations as a function of temperature.

The results of these calculations are summarized in figure 1, which shows the USF configuration Helmholtz free energies at zero pressure for the glide and shuffle sets as a function of temperature. Over the entire temperature range, the Helmholtz free energy of the glide set USF configuration is higher than for the shuffle set, although the difference between them decreases with increasing temperature. Figures 2 and 3 show the respective contributions of the enthalpy and entropy differences between the glide and shuffle USF configurations.

At this point it is interesting to analyze the results of these MD simulations within the framework of Rice's theory on dislocation nucleation. So far, the calculations have ignored the possible influence of finite pressure conditions. These effects should be taken into account in order to obtain a more realistic picture of the energetics involved in the 
dislocation nucleation criterion. To this end the introduction of the Gibbs free energy per unit area according to the definition adopted by Kaxiras and Duesbery 4 and Juan and Kaxiras 3 is appropriate:

$$
G(P, T)=F(T)+P \Delta z .
$$

Here $F(T)$ is the Helmholtz free energy per unit area (determined from the adiabatic switching simulations) and $\Delta z$ represents the volume relaxation perpendicular to the slip plane under consideration. According to the dislocation nucleation criterion, the condition that the preferred slip plane changes from shuffle to glide is given by $G_{\text {shuffle }}(P, T)=G_{\text {glide }}(P, T)$, which describes the $(P, T)$ coexistence curve separating the two dislocation nucleation regimes in the phase diagram.

Before embarking on the construction of the EDIP phase diagram, the question related to the quantitative $\gamma_{u s}$ discrepancy mentioned earlier should be addressed. According to Table if, EDIP overestimates the difference between the glide and shuffle values of $\gamma_{u s}$ at zero temperature (including atomic and volume relaxation) by more than a factor three. Since this discrepancy is reflected in the finite temperature values of $\gamma_{u s}$, it strongly affects the free energies and distorts the corresponding EDIP phase diagram. In order to eliminate this effect, the EDIP Helmholtz free energy differences are corrected in such a manner that the zero temperature value equals the corresponding DFTLDA value. This correction is most conveniently accomplished by means of a simple rigid energy shift imposed for all temperatures. Such a shift only modifies the static energy scales while it leaves unaltered the dynamical entropic properties.

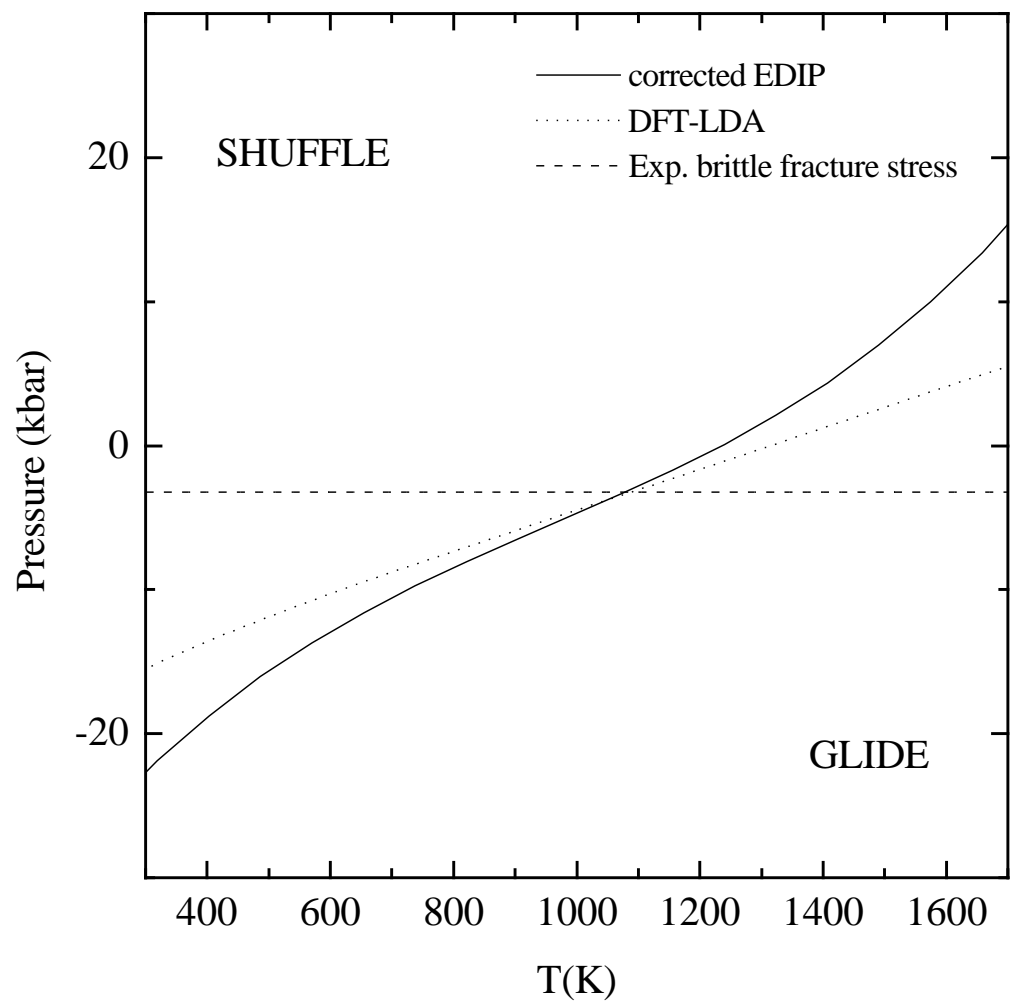

FIG. 4. Phase diagram: Coexistence curves separating the preferable nucleation of dislocations on shuffle planes versus glide set. The full line represents the results obtained with the EDIP potential after correction for the overestimated difference in $\gamma_{u s}$. The dotted line represents the DFT-LDA calculations by Juan and Kaxiras (Ref. 6). The dashed line represents the experimental brittle fracture stress. 
Figure 4 shows a comparison between the corrected EDIP phase diagram (continuous line) and the DFT-LDA results 5 (dotted line). For $(P, T)$ values below (above) these curves, the glide (shuffle) set USF configuration has lower free energy. Both coexistence curves agree fairly well, with derivatives $d P / d T$ of the same order of magnitude over the entire temperature range. Furthermore, the curves intersect near $1100 \mathrm{~K}$ with nearly equal slopes. The most significant difference between both curves is the inflection point which appears in the EDIP phase diagram and originates from the specific behavior of the glide-shuffle entropy difference as a function of temperature (negative first derivative and positive second derivative). However, despite this discrepancy, both phase diagrams show no pronounced differences. This suggests that the exact treatment of temperature dependent vibrational effects in the present simulations does not significantly alter the qualitative picture resulting from the approximate TST approach adopted in earlier DFT-LDA calculations.

The phase diagrams in figure 1 illustrate the basic idea of an abrupt transition from shuffle to glide set dominance under specific temperature and pressure conditions. Such a transition may be related to the sharp BDT transition observed in silicon. Although dislocations nucleate more easily on shuffle planes, the splitted Shockley partials on the glide set are more mobile. In this sense, the transition from brittle to ductile behavior might be directly related to the abrupt transition from shuffle to glide set dominance. Within such an interpretation, the EDIP and DFT-LDA phase diagrams might indicate a transition temperature at the intersection points of the coexistence curves with the experimental brittle fracture stress. In this manner both EDIP and DFT-LDA would predict a transition temperature of $1100 \mathrm{~K}$ which is not unreasonably far from the experimental critical temperature of $873 \mathrm{~K}$.

These results can not be taken literally for several reasons however. First, important factors such as electronic entropy contributions, surface free energies and dislocation core reconstruction effects have been neglected in the present approach. Furthermore, the microscopic mechanisms involved in the BDT transition phenomenon may involve subtleties which are not included in the approach adopted in this work. Therefore, a direct quantitative interpretation of the dislocation nucleation criterion represents an oversimplification, although we expect the qualitative picture furnished by this approach to be reasonable.

In summary, we have calculated the free energies of the shuffle and glide USF configurations in silicon as a function of temperature. For this purpose we used the recently developed empirical EDIP model and applied the MD adiabatic switching method which allows accurate and efficient determination of thermal quantities including all anharmonic effects. The results of the finite temperature MD simulations agree fairly well with earlier DFT-LDA calculations on the transition from shuffle to glide dominance as a function of temperature. This suggests that the full inclusion of finite temperature effects in our simulations does not significantly alter the qualitative picture provided by the TST DFT-LDA approach, in which such effects were treated in an approximate manner.

M.K. and A.A. acknowledge financial support from FAPESP, CAPES, FAEP, and CNPq, and J.F.J. acknowledges support from FAPESP, all of them Brazilian funding agencies. M.B. and E.K. acknowledge support by the Harvard MRSEC and J.F.J. acknowledges support by the MIT MRSEC, both of which are funded through NSF.

${ }^{1}$ J. Samuels and S.G. Roberts, Proc. R. Soc. London A 421, 1 (1989).

${ }^{2}$ P.B. Hirsch, S.G. Roberts and J. Samuels, Proc. R. Soc. London A 421, 25 (1989).

${ }^{3}$ J.P. Hirth and J. Lothe, Theory of dislocations (Wiley, New York, 1982).

${ }^{4}$ E. Kaxiras and M.S. Duesbery, Phys. Rev. Lett. 70, 3752 (1993).

${ }^{5}$ Y-M Juan and E. Kaxiras, Phil Mag. A 74, 1367 (1996).

${ }^{6}$ J.R. Rice, J. Mech. Phys. Solids 40, 239 (1992).

7 J.R. Rice and G.E. Beltz, J. Mech. Phys. Solids 42, 333 (1994).

${ }^{8}$ Y. Sun, G.E. Beltz, Mater. Sci. Eng. A170, 67 (1993).

${ }^{9}$ G.H. Vineyard, J. Phys. Chem. Solids 3, 121 (1957).

${ }^{10}$ F.H. Stillinger and T.A. Weber, Phys. Rev. B 31, 5262 (1985).

11 J. Tersoff, Phys. Rev. B 38, 9902 (1988).

${ }^{12}$ M. Z. Bazant, E. Kaxiras and J.F. Justo, Phys. Rev. B 56, 8542 (1997).

13 J.F. Justo, M. Z. Bazant, E. Kaxiras, V. Bulatov and S. Yip, to appear in Phys. Rev. B 58 (1998).

${ }^{14}$ M. Watanabe and W.P. Reinhardt, Phys. Rev. Lett. 65, 3301 (1990).

${ }^{15}$ M. de Koning and A. Antonelli, Phys. Rev. E 53, 465 (1996).

${ }^{16}$ M. de Koning and A. Antonelli, Phys. Rev. B 55, 735 (1997). 